\title{
Política de intermodalidad para la movilidad metropolitana Padre Las Casas-Temuco, sur de Chile ${ }^{*}$
}

Intermodality policy for metropolitan mobility Padre Las Casas-Temuco, southern Chile

\author{
Mario Fuentes Valenzuela** \\ Andrés Matta Cuminao*** \\ Juan Vásquez Gómez**** \\ César Villacís Arreaga*****
}

\begin{abstract}
Resumen: El paper propone una política que integre la movilidad eléctrica por trenes de cercanía como principal instrumento masivo para resolver la alta congestión en los desplazamientos entre la comuna dormitorio de Padre Las Casas en la rivera sur del Río Cautín y el centro de Temuco, capital de la Región de la Araucanía. En la centralizada política chilena se requiere acuerdos con la Empresa de Ferrocarriles del Estado (EFE) para lograr un sistema periódico y a su vez la cooperación intergubernamental del Gobierno Regional, municipios y ministerios sectoriales (Transporte, Obras Pública, Medio Ambiente y Vivienda) para lograr la colaboración y los recursos para un sistema integrado de transporte en una de las tres zonas metropolitanas del sur de Chile (junto a Concepción y Puerto Montt-Puerto Varas). También es relevante la participación ciudadana en todo el proceso para generar un proyecto que considere preferencias y comprometa el uso de una red eficiente de transporte público para contribuir a la alta contaminación del Gran Temuco
\end{abstract}

Palabras clave: Temuco, Conectividad vial, intermodalidad en transporte, políticas públicas, seguridad vial, descontaminación.

\begin{abstract}
The paper proposes a policy that integrates electric mobility by commuter trains as the main mass instrument to solve the high congestion in commuting between the dormitory commune of Padre Las Casas on the south bank of the Cautín River and the center of Temuco, capital of the Araucania region. In the centralized Chilean policy, agreements with the State Railways Company (EFE) are required to achieve a periodic system and in turn the intergovernmental cooperation of the Regional Government, municipalities and sector ministries (Transport, Public Works, Environment and Housing) to achieve collaboration and resources for an integrated transportation system in one of the three metropolitan areas in southern Chile (along with Concepción and Puerto Montt-Puerto Varas). Citizen participation is also relevant throughout the process
\end{abstract}

\footnotetext{
* Investigación en el módulo Municipio y Sociedad Política, Participación y Gobernanza - Magíster Gestión Pública Municipal y Desarrollo Local, Universidad Autónoma de Chile, sede Temuco.

** Profesor de Educación Física, Universidad San Sebastián, Concepción, mariofuen18@gmail.com

*** Ingeniero Agrónomo, Universidad de La Frontera, sede Temuco, mattacuminao@gmail.com

**** Profesor Educación Media, Universidad de Los Lagos, Osorno, juanfvasquezg@gmail.com

***** Licenciado en Administración Pública, Universidad Península de Santa Elena. (Ecuador), c.villacis.sep64@gmail.com
} 


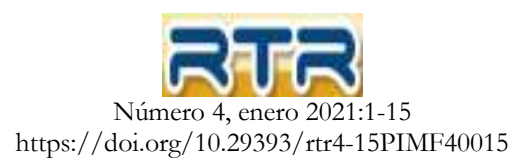

to generate a project that considers preferences and compromises the use of an efficient public transport network to contribute to the high pollution of Greater Temuco.

Keywords: Temuco, Road connectivity, intermodality in transport, public policies, road safety, decontamination.

Recibido: 14 octubre 2020 Aceptado: 19 diciembre 2020

\section{Introducción}

La comuna de Temuco a través del trabajo de su municipio y el Instituto de Desarrollo Local y Regional (IDER) de la Universidad de La Frontera, ha logrado conocer de forma directa las reales necesidades de cada sector urbano y rural, identificando múltiples desafíos, en donde la ciudadanía ha puesto importantes retos, principalmente, en los temas medioambientales, seguridad pública y conectividad, prevaleciendo la interacción con la ciudadanía en pos de la construcción de una ciudad ordenada y amigable con el medio ambiente, en donde todos y todas puedan convivir de manera segura y transitar por vías y espacios públicos pensados en las personas y sus familias.

La actividad comercial de la ciudad, concentrada en mayor grado en el centro o área fundacional y en un área de influencia próxima, atrae una alta población flotante cuya motivación de viaje a la capital regional está originada por el acceso a educación, comercio,

servicio, salud, empleo, convirtiendo de esta forma a la ciudad en el principal centro de intercambio económico, de información y tecnología de la región.

El alto flujo asociado al Hospital Regional "Hernán Henríquez" único centro de salud de Nivel 1 en la región, abastece a la población de todas las comunas, las que acceden principalmente desde el transporte público (urbano e inter urbano), concentrando un alto flujo de peatones en las intersecciones de las calles Montt y Caupolicán, donde según el conteo realizado por SECTRA en el año 2017 esa intercesión concentró la mayor cantidad de peatones de la ciudad llegando a un pick de 4.416 en hora punta mañana. Actualmente, se registran 10 Universidades, 8 Institutos Profesionales y 5 Centros de Formación Técnica en la ciudad, concentrando servicios de educación especializados y atrayendo gran cantidad de población joven. Por las características antes descritas, las actividades que se dan en la ciudad son de carácter principalmente terciario y en menor medida secundario. En este sentido, los principales sectores económicos de la comuna de acuerdo al número de empresas, ventas generadas y trabajadores dependientes contratados, corresponde a Comercio, Construcción, Actividades inmobiliarias, empresariales y de alquiler, Servicios Sociales y de Salud y Enseñanza.

En la actualidad, la conurbación de Temuco y Padre Las Casas se ubican en la sexta área urbana de Chile por cantidad de habitantes, debiendo enfrentar diversas funciones específicas como centro urbano de mayor complejidad en la región, identificando su función administrativa de capital regional y provincial, generando la localización de las sedes regionales de todas las dependencias públicas, propiciando un uso de suelo asociado a servicios de alta especificidad, una alta atracción de viajes y población desde las inter comunas hacia Temuco, tanto para acceder a estos servicios, así como fuente laboral.

La historia de Padre las Casas comienza a escribirse años antes de la ocupación de La Araucanía, cuando llegan a esta región unos comerciantes ambulantes que se establecen en los faldeos del Cerro Conunhuenu y en las indefensas riberas del veleidoso torrente del Cautín, dando nacimiento a 


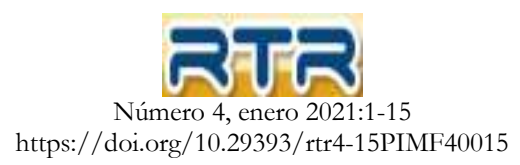

Villa Alegre, nombre con el que en la actualidad se conoce su principal arteria. Esta tierra, en sus tiempos de origen, asolada por bandoleros y plagada de cantinas, escenario de sangrientas batallas, lleva el nombre del predicador español, Fray Bartolomé de las Casas, llamado "El Apóstol de los Indios" por su labor evangelizadora con los indios de Cuba, México y Guatemala.

En cuanto a la accesibilidad hacia la capital regional, esta presenta importantes limitaciones para un adecuado flujo vehicular, ya que solo se puede acceder a través de dos únicas vías. Una que permite una salida más directa hacia zona sur del país y que después empalma con la carretera 5-Sur. Esta vía también permite el acceso a Temuco sur, pero no se accede directamente al distrito central y de servicios. La otra ruta de acceso es la que cruza sobre el río Cautín. Aunque es una vía directa al centro de Temuco, en las horas de mayor flujo vehicular, la circulación es lenta, situación que se hace más crítica cuando este acceso es utilizado por vehículos de tracción animal provenientes del sector rural, especialmente del sector Truf-Truf-Niagara (nororiental de la comuna).

Como resultado de la implementación en un período a corto y mediano plazo de esta política pública entre las comunas de Temuco y Padre Las Casas, se logrará sustentar el resultado esperado en la conectividad vial, logrando la consecución de los objetivos planteados como mejorar el flujo y disminuir el tiempo de desplazamiento entre ambas comunas, factor de suma relevancia frente a la gran necesidad de traslado de la ciudadanía.

\section{Desarrollo}

Es por estos antecedentes, que resulta imprescindible la construcción de un diseño moderno de conectividad vial entre ambas comunas, con infraestructura acorde a las necesidades actuales y con una tecnología de punta, conservando los siguientes parámetros:

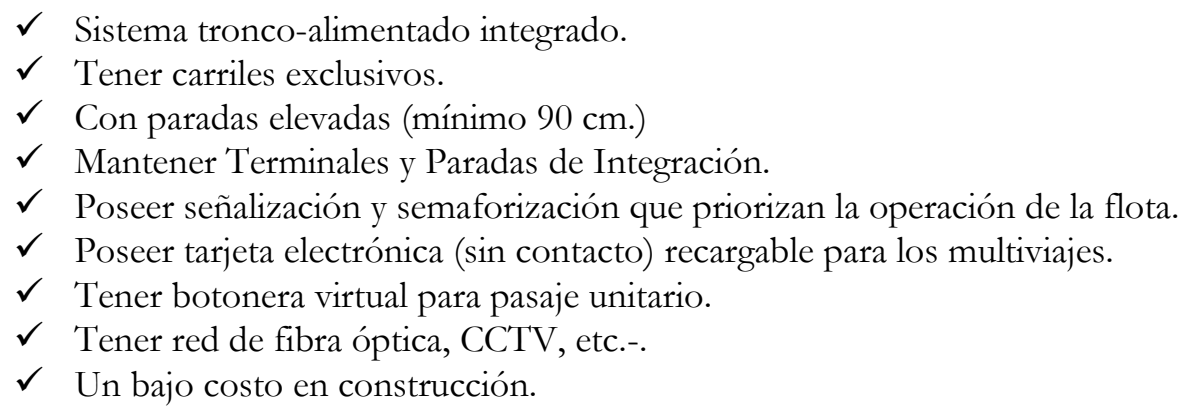

"Temuco, es el Centro Regional de servicios y comercio de La Araucanía; reconocida como una comuna inclusiva, responsable con el medio ambiente, con una movilidad urbana y rural acorde a los requerimientos de las personas y sus barrios, posee espacios públicos que favorecen la calidad de vida de sus habitantes, promoviendo la interculturalidad como parte de su identidad" (PLADECO 2020-2024).

\section{Diagnóstico Comunal}

La comuna de Temuco se ubica en la región de La Araucanía y pertenece a la provincia de Cautín, comprende una superficie de $464 \mathrm{~km} 2$ de superficie, que representa el 1,5\% de la superficie de la región. Con una población de 282.415 habitantes (de acuerdo a los resultados del Censo del año 


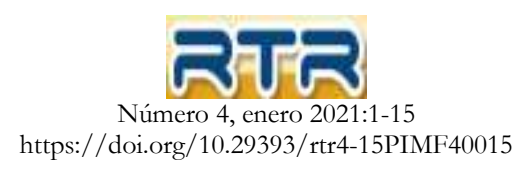

2017) es la comuna con mayor cantidad de población a nivel regional, luego la siguen las comunas de Padre Las Casas, Villarrica, Angol y Lautaro.

Conforme a su ubicación geográfica, limita al Norte con la comuna de Galvarino; al Noreste con la comuna de Lautaro; al Este con la comuna de Vilcún, al Sur con la comuna de Padre Las Casas y al Oeste con las comunas de Cholchol y Nueva Imperial.

La comuna de Padre Las Casas se sitúa en el centro de la Región de La Araucanía, entre las comunas de Temuco, Nueva Imperial, Freire y Vilcún y en el sector más occidental de la Asociación de Municipios de la Precordillera (AMP). Tiene una superficie de 400,7 Km2 y 58.795 habitantes, la densidad poblacional es de 147 habitantes por km2. (INE, 2002).

Es una comuna mesopotámica, dado que tiene como límite norte el río Cautín y como límite sur el río Quepe y su afluente Huichahue.

\section{Diagnóstico del Transporte Público en la Capital de la Araucanía}

En función a la recopilación de antecedentes de organismos públicos, podemos constatar que desde la década recién pasada se viene poniendo en práctica instrumentos de planificación que tienen como finalidad, dar solución a las necesidades de mejora y modernizar del transporte público para la ciudad de Temuco y Padre Las Casas. Es así como se crea la resolución $\mathrm{N}^{\circ} 161$, del ministerio de transporte y telecomunicaciones, la cual establece un plan de modernización al transporte público entre Temuco y Padre Las Casas, como también vía exclusiva en la ciudad de Temuco para la circulación de vehículos que indica dicho texto normativo.

\section{“Considerando":}

1.- Que, según estudios técnicos efectuados por el Área de Transporte Público Regional, en adelante, TPR Araucanía, perteneciente a la Secretaría Regional Ministerial de Transportes y Telecomunicaciones de la Región de la Araucanía, a través del informe técnico "Diseño de Vías Exclusivas y Punto Intermodal Temuco", desarrollado por Citra Limitada, justifica la implementación de Vías Exclusivas en la calle Portales, en la comuna de Temuco, como parte de medidas de gestión de tránsito que prioricen el bien común, propendiendo a entregar un transporte público de calidad.

2.- De acuerdo a lo anterior, esta Secretaría Regional Ministerial se encuentra implementando un Plan de Modernización al Transporte Público, el que busca mejorar el estándar del Sistema de Transporte Público de la conurbación Temuco - Padre Las Casas. Para lo anterior, es de fundamental relevancia, complementar las futuras mejoras operacionales, con la implementación de medidas de gestión de tránsito e infraestructura, tales como vías con preferencia o exclusividad para la circulación de Transporte Público, contribuyendo a la reducción de tiempos de viaje del Transporte Público Urbano de la ciudad de Temuco.

3.- Que, el DFL $\mathrm{N}^{\circ} 1$, de 2007 , citado en vistos, en su artículo $2^{\circ}$, numeral 48, define como Vía exclusiva a la calzada debidamente señalizada, destinada únicamente al uso de ciertos vehículos, determinados por la autoridad competente. Por su parte, el artículo 113 de esta última norma dispone que el Ministerio de Transportes y Telecomunicaciones podrá prohibir, por causa justificada la circulación de todo vehículo o de tipos específicos de éstos, por determinadas vías públicas, facultad que ha sido delegada en las Secretarías Regionales del ramo mediante resolución $\mathrm{N}^{\circ}$ 59, de 1985, del 


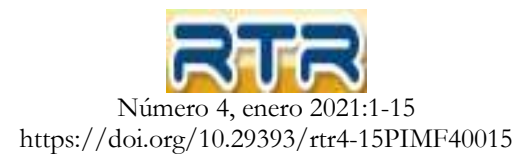

signado Ministerio. En consecuencia, conforme a los estudios y disposiciones legales previamente anotadas, esta Secretaría Regional se encuentra facultada para resolver lo que a continuación se indica:

\section{Resuelve:}

1.- Establécese como medida de gestión de tránsito en la conurbación de Temuco - Padre Las Casas, de lunes a viernes, excepto festivos, de 07:00 horas a 20:00 horas, según sentido de circulación, vía exclusiva con prioridad para los vehículos de transporte público de pasajeros que se indica en el resuelvo 2, en la vía y tramo que a continuación se indica:

Eje Portales

\begin{tabular}{|c|c|c|c|}
\hline Vía & Desde & Hasta & $\begin{array}{c}\text { Sentido Vía } \\
\text { Exclusiva }\end{array}$ \\
\hline Portales & Vicuña Mackenna & Av. Barros Arana & $\begin{array}{c}\text { Habitual, Poniente - } \\
\text { Oriente }\end{array}$ \\
\hline
\end{tabular}

2.- Sólo podrán circular por estas vías exclusivas, los siguientes vehículos:

a) Buses de servicios de transporte público urbano de pasajeros, que se encuentren inscritos y prestando servicios en el Registro Nacional de Servicios de Transporte Público de Pasajeros.

b) Vehículos inscritos en la modalidad de Taxi colectivo que se encuentren prestando servicio en el Registro Nacional de Servicios de Transporte Público de Pasajeros, exclusivamente en el tramo inscrito en el certificado de recorrido.

3.- Además, de forma excepcional podrán transitar los siguientes vehículos: servicios de emergencia (Bomberos, Carabineros, ambulancias, etc.), del Programa de Fiscalización de la Subsecretaría de Transportes y de los municipios, destinados a cumplir funciones de seguridad ciudadana, debidamente identificados como tales. Adicionalmente, los vehículos no contemplados en los párrafos precedentes y que deban necesariamente utilizar esta vía exclusiva, con el objeto de ingresar o egresar desde sus lugares de residencia o estacionamiento habitual podrán hacerlo siempre y cuando lo realicen por un trayecto menor o igual a 2 cuadras.

4.- El presente acto administrativo entrará en vigencia a contar del jueves 1 de marzo de 2018.

5.- Carabineros de Chile, inspectores municipales e inspectores del Ministerio de Transportes y Telecomunicaciones, son los encargados de fiscalizar el estricto cumplimiento de la medida antes señalada de conformidad al DFL No 1 , de 2007, ya citado"

Por otra parte la SEREMI de Transporte y Telecomunicaciones de la Araucanía, ha levantado un Mapa Estratégico de desarrollo el cual se traduce en mejorar la calidad de vida de los habitantes alcanzando una movilidad con equidad, de calidad y eficiente. De manera paralela el presente mapa hace sentido respecto a nuestra política dado a que se encausa en una oferta de infraestructura adecuada al desarrollo y de largo plazo. 


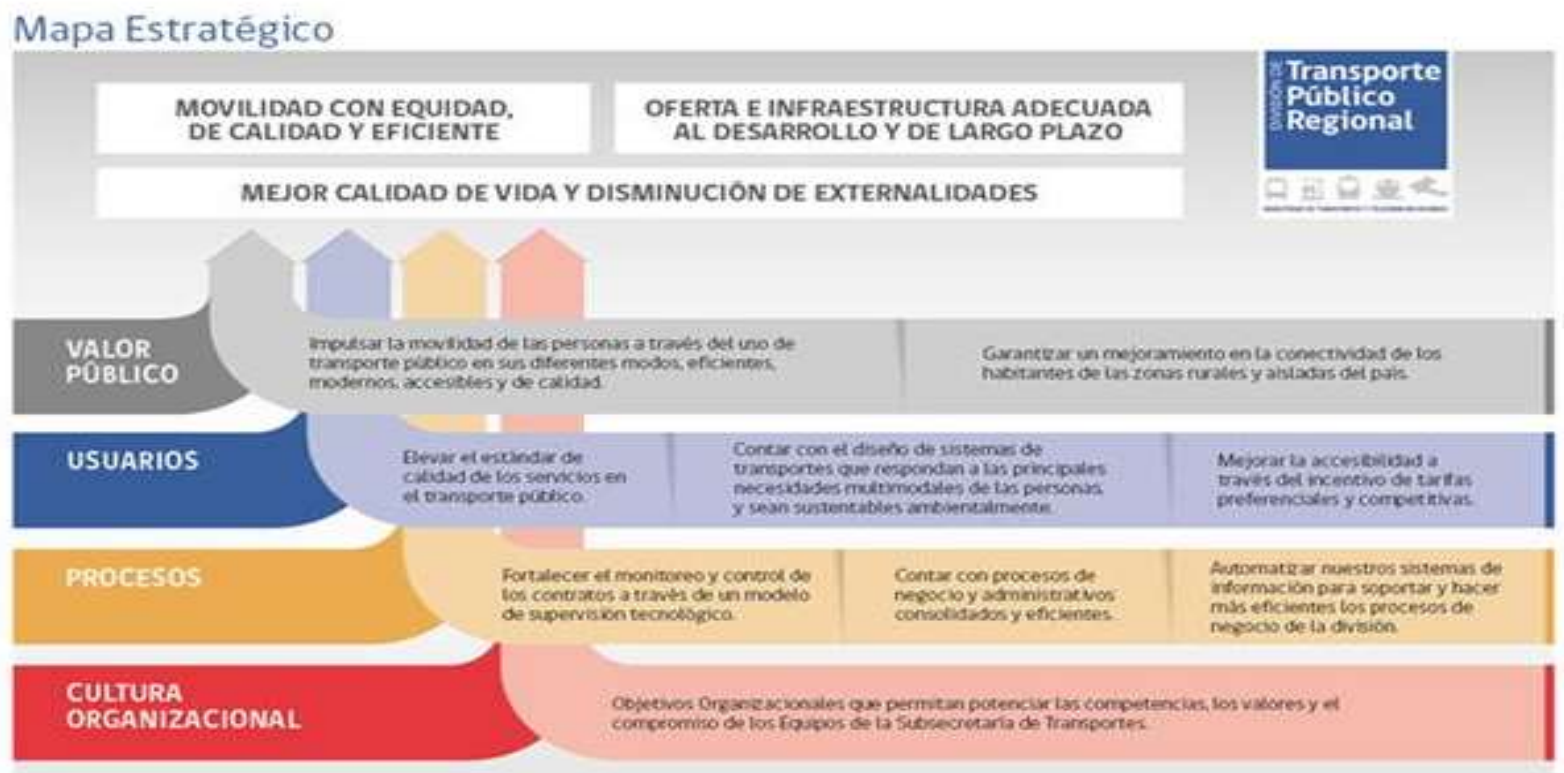

\section{Municipios, construcción de redes asociativas comunitarias}

Proyectos de tal envergadura como la planteada en la presente Política de Conectividad Vial, requiere de la toma de decisiones y compromiso presupuestario del Estado, es decir desde el nivel central, por cuanto, comprendiendo el escaso grado de incidencia que poseen los municipios en la toma de decisiones ministeriales, se requiere de un tratamiento estratégico y participativo, no basta con una solicitud y acuerdos de voluntades entre ambos municipios involucrados, sino, un trabajo asociativo multi sectorial que confluya a la creación y articulación de una red de actores relevantes involucrados .

En este ámbito, vemos que las facultades de los municipios son acotadas y en esta materia el cuerpo legal que las rige; Ley Orgánica Constitucional de Municipalidades $N^{\circ} 18.695$, al respecto señala lo siguiente:

Artículo $4^{\circ}$.- Las municipalidades, en el ámbito de su territorio, podrán desarrollar, directamente o con otros órganos de la Administración del Estado, funciones relacionadas con:

\section{h) “El Transporte y Tránsito Públicos".}

Al tenor de lo expuesto, es que la presente Política de Conectividad Vial, propone la creación de un Comité de Adelanto, que busque la canalización de la sinergia emanada de diversos actores comunitarios que se verán beneficiados, de manera tal, de incidir y formar parte de los diversos instrumentos de planificación, tanto de nivel municipal como ministerial, a fin de converger en una política altamente participativa y de representatividad ciudadana.

En el texto "Adiós al municipio o la fecundidad de una red de Gobierno Local" (1998), Esteban Valenzuela señala que:

"Municipio con mayores poderes es hoy utópico en un sistema donde el gobierno central y las oficinas desconcentradas de los ministerios tienen el gran poder. ¿Qué hacer, entonces, ante la falta de recursos y 


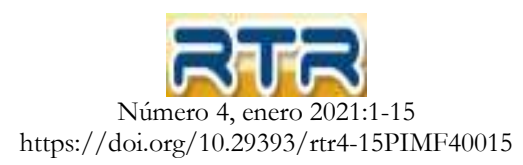

de poder en áreas claves del desarrollo comunal? La solución es constituir esta red invisible e informal, que es el verdadero gobierno de la comuna. Un ámbito de cooperación inter-institucional, que movilice recursos de fuentes públicas y privadas, para así salir del lamento y encabezar un proceso de desarrollo local. El poder no está en ninguna parte y está en todas partes. La ventaja para encabezar este proceso la tiene el municipio, por su cercanía a la gente y la legitimidad que da el ser elegido directamente por el pueblo. Ese poder debe ser usado para articular esta red de gobierno local, donde el municipio cobre vigencia y fecundidad si se abre a esta multiplicidad de cooperación. El municipio como gobierno local está muerto en Chile, pero vive en la posibilidad de liderar a los actores públicos, privados y sociales de su territorio".

"Decimos adiós al municipio porque el siglo XXI, con sus procesos de globalización y el surgimiento cada vez más masivo de formas de hacer política y participar, hará perder centralidad a la forma tradicional de valorar el Estado y los propios municipios. Cada vez más los actores del territorio serán diversos y con distintos niveles de poder y gobernabilidad: redes de cooperación entre instituciones públicas, corporaciones de desarrollo, municipios que territorializan su acción, convenciones intermunicipales, agencias internacionales, fundaciones privadas, organizaciones comunitarias y ONG, consejos sociales amplios... Este cambio histórico es ineludible. Lo que perdura es la necesidad de que "alguien gobierne lo que es común, lo que es ciudadano". Pero quizás ya no será la idea de ciudad griega, sino una comunidad que gestiona un colegio, consejos vecinales que resuelven el presupuesto municipal territorializado de una comuna, consejos amplios que fijan los fondos para la tercera edad o la cultura, corporaciones privadas que se hacen cargo del desarrollo industrial y la capacitación, policías mixtas de tipo central y local. El gobierno tiene distintos dominios y crecerá la autogestión." Seguridad y Medio Ambiente.

\section{Seguridad y Transporte}

Al hablar de la mejora de los estándares de calidad de vida, sin lugar a duda el factor "seguridad" juega un rol relevante, especialmente cuando este último concepto está ligado a los indicadores de accidentes, es por ello, que el tren se vuelve otra manera de viajar muy apreciada, ya no solo por la nostalgia de los románticos viajeros, sino, porque el tren sigue siendo ese medio de transporte que ha mejorado mucho en cuestión de seguridad.

Lema de la Política: "Conectividad Vial Segura y Expedita"

\section{Objetivo General:}

- Generar, promover y potenciar de forma participativa el desarrollo de la conectividad vial entre las comunas de Temuco y Padre Las Casas, región de La Araucanía.

\section{Objetivos Complementarios:}

- Establecer con la ejecución de esta Política Pública la reducción de gases contaminantes, que permita un desarrollo sustentable en las comunas de Temuco y Padre Las Casas.

- Promover el progreso de conectividad vial con actores público privados de las comunas y de la región.

- Disminuir los tiempos de traslados de las personas con este proceso local vinculante dentro de su territorio. 


\section{Indicadores de la política pública:}

Estos indicadores permitirán establecer que se cumplan de manera eficiente y eficaz por parte de la comisión que revisara la política pública a la cual nos estamos refiriendo, y los principales indicadores serán:

- Disminuir los tiempos de traslado entre las comunas de Temuco y Padre las Casas, disminución a un $50 \%$ en un lapso de 1 año desde la implementación de esta política de interconexión vial.

- Disminución de gases contaminantes, considerado que Temuco y Padre las Casas son las ciudades del sur de Chile más contaminadas, bajar índices en 60\% en un Lapsus de 2 años.

- Disminución de accidentes de tránsito en las principales avenidas como por ejemplo, Avda. Caupolicán, Avda. Alemania, etc.; bajar índices 40\% en un período de 2 años.

- Incentivar el uso del transporte público, trenes entre Temuco y padre las casas.

- Seguridad y bienestar Público, es posible mediante buses y trenes nuevos con mayor capacidad dotados de cámaras de seguridad, aire acondicionado etc.; lo que posteriormente disminuirán los estados de stress.

Indicadores complementarios de la Política Pública: De las ideas expuestas en los párrafos anteriores, se proponen indicadores complementarios, siendo los siguientes:

- Dotar a Temuco terminales integrados a las afuera de los sectores urbanos, para así evitar congestión en el sector de Feria Pinto, calle Balmaceda dentro de un plazo de 3 años

- Realizar talleres educativos e informativos sobre la implementación de política pública.

- Reducción de tarifas a adultos mayores y estudiantes.

- Construcción de red de ciclovías en las principales vías Temuco y Padre Las Casas en un lapsus de 3 años.

- Sistema de semáforos automatizados controle el tránsito y el transporte público.

- Automatización electrónica integrada de pago de boletos que permita viajar por los distintos lugares de la Red Vial Intercomunal.

Para dar cumplimiento a esta política pública, dentro de los objetivos secundarios, será propuesto que, en un lapso de 3 años dotar de mayores ciclovías a la comuna. Para llevar a cabo este punto se presenta una calendarización de actividades para concretar tal objetivo:

\begin{tabular}{|l|c|c|c|}
\hline \multicolumn{1}{|c|}{ Calendarización } & Año 1 & Año 2 & Año 3 \\
\hline Diseño y búsqueda de Recursos Públicos. & $\mathrm{X}$ & & \\
\hline $\begin{array}{l}\text { Incorporación de esta Política Pública a los Planes de Desarrollo } \\
\text { Comunal. }\end{array}$ & $\mathrm{X}$ & & \\
\hline $\begin{array}{l}\text { Reuniones de planificación con el Ministerio de Transporte } \\
\text { Creación de la mesa sectorial u organismo técnico para aplicar la } \\
\text { Política Pública. }\end{array}$ & $\mathrm{X}$ & $\mathrm{X}$ & $\mathrm{X}$ \\
\hline $\begin{array}{l}\text { Reuniones semestrales para revisión de estados de avance de la } \\
\text { implementación de la Política Pública. }\end{array}$ & $\mathrm{X}$ & $\mathrm{X}$ & $\mathrm{X}$ \\
\hline
\end{tabular}




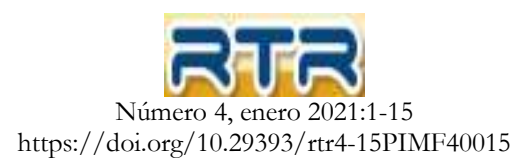

Para que esta política pública pueda concretarse en el territorio es preciso crear una corporación para el adelanto de la movilidad vial, con un plebiscito vinculante como mecanismo de consulta, en donde las personas y actores sociales participen activamente en el diseño, implementación, ejecución y evaluación del proyecto. Para que esta política pública pueda ejecutarse correctamente enumeraremos a los actores involucrados en el siguiente listado:

- La Secretaría Comunal de Planificación (SECPLA) Temuco, Mauricio Reyes Jiménez

- Ministerio de Obras Públicas Araucanía, Rodrigo Tolego

- Región de la Araucanía Seremi de Transporte: Luis Calderón Ramírez

- Asociación Gremial dueños de buses urbanos de la ciudad de Temuco, Juan Araneda

- Sindicato de Choferes de buses Temuco-Padres las Casas

- Unión comunal juntas de vecinos urbanas Araucanía, Mariela Nancy Loncoman Rodríguez

- Ministerio de Bienes Nacionales de la Araucanía, Natalia Rivera

- Alcalde de Temuco, Sr. Jaime Salinas Mansilla

- Alcalde de Padre las Casas, Sr. Juan Eduardo Delgado Castro.

- Los Concejales de Temuco: Sra. Solange Carmine Rojas, Sr. Pedro Durán Sanhueza, Sra. Constanza Saffirio López, Sr Roberto Neira Aburto, Sr Oscar Albornoz Torres, Sr Jaime Salinas Mansilla, Sr Marcelo León Acevedo, Sr Alejandro Bizama Tiznado, Sr Esteban Barriga Rosales, Sr José Luis Velasco Guzmán.

- Los Concejales de Padre Las Casas: Sr. Raúl Henríquez Burgos, Sra. Evelyn Mora Gallegos, Sr. Alex Henríquez Araneda, Sr. Juan Francisco Nahuelpi Ramírez, Sr. Pedro Vergara Manríquez, Sr. Jaime Catriel Quidequeo.

- Gobernador de Cautín, Richard Caifal

- Cámara de Comercio Servicios y Turismo de Temuco A.G, Presidente: Gustavo Valenzuela Ibáñez.

- Cuerpo de Bomberos, Comandante Claudio Magofke Rodríguez

- Centros de Alumnos de Liceos de la Comuna. (Diurnos y nocturnos).

\section{Mecanismo de Participación:}

La participación ciudadana multiactor desde su conformación del "Consejo de Planificación Estratégica”; básicamente se conforma con los actores antes mencionados, quienes serán aportes por su conocimiento del territorio para la consecución de la Política Pública de Conectividad Vial, en beneficio de ambas comunas. La participación pasa a ser un indicador, donde la propuesta se valida entre los distintos actores para así poder ser presentada en el mundo público y privado para su financiamiento.

\section{Participación multiactor:}

Es relevante, involucrar a los actores débiles en estas consultas vinculantes, donde la participación para orientar sea de suma importancia en varios puntos; como lo es, informar, consultar, colaborar, empoderar e involucrar; donde cada una de ellas juegan un rol trascendental al ser ejecutada en los actores involucrados, delegando ciertos espacios, en el cual se toman decisiones conjuntas para admitir propuestas. 


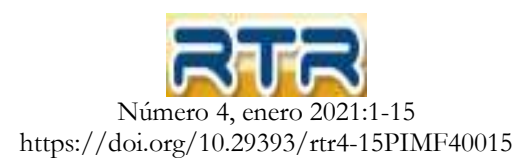

A continuación, ponemos en conocimiento varias experiencias exitosas de Políticas Públicas de conectividad vial desarrolladas en el Chile e Internacionalmente, con resultados fundamentales para mejorar los servicios de transporte en la comunidad y los usuarios afrontan diariamente este medio para sus traslados.

\section{Experiencia exitosa, CURITIBA, PARANA, BRASIL}

Durante las décadas de los 50 a los 90, las ciudades brasileñas sufrieron un intenso proceso de urbanización. Curitiba, capital del Estado de Paraná, presentó una de las mayores tasas de crecimiento anual comparativamente a otras capitales brasileñas. En la década de los 60, tenía 350.000 habitantes y una tasa de crecimiento anual alrededor de 5\%. Dicho crecimiento se debió principalmente a la mecanización del campo en el interior del Estado, generando una gran liberación de mano de obra, que salió de las zonas rurales y pequeñas ciudades en búsqueda de mejores condiciones de vida y de trabajo. La capital y su Área Metropolitana, conformada por 26 municipios, absorbió migraciones de dentro del Estado de Paraná. Actualmente, la población de la ciudad es de 1.727.010 y del Ârea

Metropolitana, 3.110.455. La principal característica de este fuerte crecimiento es la ocupación acelerada de los municipios limítrofes a Curitiba, comprometiendo áreas verdes y de manantiales, además de aumentar las demandas por implantación de infraestructura y por la generación de trabajo y renta. Curitiba, sin embargo, se ha construido en forma diferente a lo largo de los últimos 40 años. La planificación de un nuevo modelo de expansión urbana A partir de 1965, con la elaboración del Plan Preliminar de Urbanismo la creación del IPPUC - Instituto de Pesquisa e Planeamiento Urbano de Curitiba y, en 1966, la aprobación del Plan Regulador, el transporte, la circulación vial, el trabajo, la recreación, la promoción social y la vivienda, pasaron a ser pensados dentro de una visión integrada de ciudad con los principales Hitos:

- La evolución del transporte colectivo y la integración metropolitana: el Eje Metropolitano de Transporte

- Uso y Ocupación del Suelo

- Terminales de Integración y Estaciones de Embarque y Desembarque

- La gestión urbana. (Pinheiro, 2005)

\section{Experiencia Exitosa BOGOTÁ, COLOMBIA 2000}

El sistema de Bogotá se ha denominado el único real BRT completo (o full BRT) que existe, debido a su amplia cobertura y sus características de servicio. A la fecha, TransMilenio tiene $84 \mathrm{~km}$ en operación y transporta alrededor de 1,4 millones de pasajeros al día. Su capacidad ha alcanzado hasta $40 \mathrm{mil}$ pasajeros/hora/sentido, lo cual es superior a una gran cantidad de sistemas férreos del mundo, aunque de la misma manera que en éstos, se ha logrado con unas tasas de ocupación de los vehículos de 6 personas por metro cuadrado. Este sistema ha sido asociado fuertemente al alcalde que estuvo a cargo de su diseño y construcción inicial (Enrique Peñalosa), quien también lo complementó con otros desarrollos como construcción de parques, infraestructura para bicicletas y peatones y otras obras de gran envergadura para la ciudad.

Un aspecto adicional de este sistema es su reciente integración con transporte no motorizado (ciclo parqueaderos en los portales), lo cual ha incrementado el área de captación del sistema 


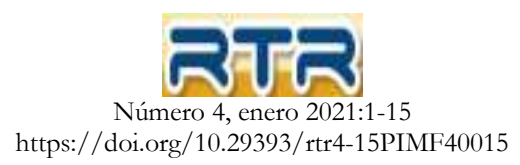

considerablemente y ha reducido la carga sobre el servicio de alimentación, que a su vez es subsidiado por la tarifa. Este sistema actualmente está en la planificación de su fase 3, y los costos capitales del sistema (incluyendo expropiación de terrenos) ha sido entre 5.3 y 13.3 millones de dólares americanos por kilómetro. Esta cifra es similar a la de algunos sistemas férreos de mediana capacidad como un tren ligero, aunque es importante un análisis más profundo para realizar estas comparaciones.

El sistema de Bogotá ha tenido grandes innovaciones en su planificación que lo han hecho el más robusto de los BRTs del mundo. Entre las características que han hecho esto algo posible están el carril de sobrepaso y los servicios expresos (que no se detienen en todas las estaciones), lo cual hizo posible tanto la reducción de tiempos de desplazamiento como el incremento de capacidad por hora por dirección. A su vez, esto y la existencia de la planificación de frecuencias de buses con base en un estándar de 6 personas por metro cuadrado, han generado algunos problemas de aglomeración en estaciones que han reducido la calidad del servicio y han incrementado la insatisfacción del usuario. (Pardo, 2009).

\section{Experiencia Valparaíso}

Metro Valparaíso transporta en promedio 45.000 pasajeros diarios en modo puro, constituyéndose la estación Limache en la quinta estación de mayor afluencia, en este modo, en la red de 20 estaciones. La estación de Limache, a su vez, registra afluencia promedio, en modo puro, de 6.000 pasajeros diarios. La operación para el transporte de pasajeros se basa en movilización por intervalos los cuales van desde 6 minutos, en horas punta en días hábiles, hasta 18 minutos, los días Domingo. La explotación comercial opera sobre la base de dos bucles, con distancia aproximada de $30 \mathrm{~km}$ para el bucle corto: Estaciones Puerto - Sargento Aldea y de $43 \mathrm{~km}$ para el bucle largo: Estaciones Puerto Limache.

Para el bucle Puerto - Limache la explotación comercial se realiza en intervalos de 12 minutos de lunes a sábado. Toda la red opera en el bucle largo (Puerto - Limache) los días Sábado y Domingo.

La operación completa, para el transporte de pasajeros, involucra una multiplicidad de sistemas y equipamiento que imponen una serie de condiciones de seguridad y secuencias operativas que permiten, en condiciones normales, sincronismo, regularidad y confiabilidad tanto en la movilización de trenes como en la gestión comercial asociada a pasajeros. Dentro de estos sistemas se menciona los más relevantes para efectos de su incidencia en un modelo de integración:

Sistema de Peaje: Sistema de registro y control de pasajeros que acceden a la red y que opera en forma distribuida en cada estación, sobre una plataforma de supervisión y control centralizada.

Sistema de Control de Tráfico: Sistema diseñado bajo el concepto de seguridad intrínseca.

Material Rodante: Flota compuesta de 27 unidades Xtrapolis 100, de fabricación francesa (2005, Alstom), donde cada unidad consta de 2 coches acoplados.

Los objetivos estratégicos de Metro Valparaíso se fijaron sobre la base de dos conceptos que constituyen los pilares de su desarrollo: Servicio e Integración. Así, la búsqueda de modelos de negocio que permitiesen subsanar el déficit de demanda estructural producto de la no implementación de un plan de transporte público integrado en la región, con Metro Valparaíso como eje, se canaliza a través de los conceptos estratégicos ya señalados. (Metro Valparaíso S.A., 2009). 


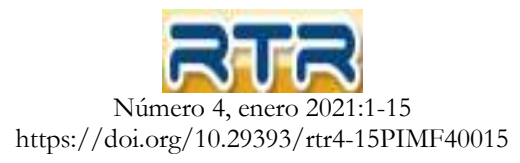

\section{Experiencia Gran Concepción Bio Tren}

El Presidente de la República y la Ministra de Transporte y Telecomunicaciones anunciaron la semana pasada la implementación del "estándar RED” en el transporte público de regiones, partiendo por nuestro Gran Concepción como la primera ciudad después de Santiago. En términos concretos, el anuncio se traduce en una flota de 25 buses eléctricos en dos recorridos que conectarán con el Biotrén en una estación intermodal del centro de la ciudad. Uno de los trazados tendrá como destino el sector Nonguén, recorriendo zonas cercanas a comercio, universidades, hospitales, el estadio regional y el terminal de buses. El otro recorrido se dirigirá por Avda. Paicaví en dirección a Talcahuano, pasando por varias casas de estudio, sectores residenciales, centros comerciales y las inmediaciones del aeropuerto Carriel Sur.

Estas son, sin duda alguna, buenas noticias. Nuestra ciudad por fin se suma a la "electromovilidad", dándole la bienvenida a un modo de transporte sustentable, moderno, cómodo y de alta calidad. Además de cuidar el medioambiente, su operación es más barata: cifras preliminares muestran que sus costos se reducen en un $70 \%$ con respecto a los de buses convencionales. Destaco también lo más importante: esta flota de buses se traduce en mayor dignidad para el usuario. Los buses tendrán asientos acolchados, aire acondicionado, wifi, cargador de celular USB y reducción significativa del ruido. Estamos a punto de recibir un nuevo modo de transporte que no sólo es mejor a lo que estamos acostumbrados, además se siente mejor.

Sin embargo, la llegada de esta nueva flota también significa importantes desafíos para nuestra ciudad. Si bien uno de sus recorridos tiene como eje Paicaví, la cual ya cuenta con un corredor de buses, el otro recorrido usa principalmente avenida Collao, una vía donde el transporte público pelea día a día por un espacio dentro del atochamiento vial. ¿Servirá de algo tener los mejores buses y experiencia de usuario si no somos capaces de ofrecer tiempos de viajes razonables? No es sólo el bus lo que debemos mejorar, también sus movimientos en nuestras vías. Ya sea con corredores nuevos o fiscalizando el correcto uso de los ya existentes. Facilitar la operación de esta nueva flota debería ser una obligación imperiosa para nuestras autoridades.

Por otro lado, ninguna ciudad del mundo ha solucionado sus problemas de movilidad con un sólo modo de transporte. Debemos abogar por la multimodalidad y conectar los buses eléctricos no sólo con el Biotren, también con modos de transporte activos como la bicicleta. No es descabellado pensar en estacionamientos de bicicletas gratuitos en las cercanías de las paradas o incluso racks (o similares) que permitan llevar la bicicleta junto con el bus.

Es necesario mencionar que, además de las condiciones de los buses, su espacio necesario en las calles y su interacción con otros modos, un elemento extra a considerar son los paraderos. De nada sirve proveer el mejor servicio dentro del vehículo si no podemos también, ofrecer una espera segura, confiable (con tiempos de llegada y frecuencias conocidos de antemano) y cómoda, incluso en condiciones meteorológicas adversas. Como las existentes en el Gran Concepción. (Espinoza, 2009, p.14).

\section{Principales fuentes de financiamiento para implementar la Política Pública}




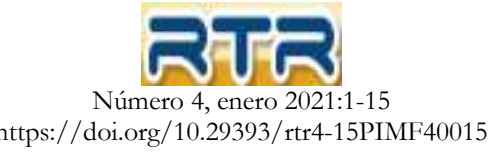

Proyectos de inversión: Se ejecutará la construcción de la primera etapa de conectividad vial entre las comunas de Temuco y Padre Las Casas; con una distancia de $5 \mathrm{~km}$, cuyo valor aproximado es de $\$ 70$ millones de pesos chilenos por kilómetro

\section{Inversión en Infraestructura de la Fase I:}

Carriles exclusivos $(5 \mathrm{~km})$

$\$ 7.000 \mathrm{M}$

Terminales (2)

$\$ 300 \mathrm{M}$

Paradas (5)

Señalización y Semaforización

Red de Fibra Óptica

TOTAL (INFRAESTRUCTURA)

Buses Articulados $4 \times \$ 200 \mathrm{M}=\quad \$ 800 \mathrm{M}$

$\$ 30 \mathrm{M}$

$\$ 15 \mathrm{M}$

$\$ 7.445 \mathrm{M}$

Buses Alimentadores $2 \times \$ 80 \mathrm{M}=\$ 160 \mathrm{M}$

TOTAL (BUSES)

INVERSION TOTAL

\section{Diseño:}

- Cámaras de seguridad en estaciones y terminales.

- Flota de ómnibus equipada con comunicación 3G y GPS Conectividad

- Circuito cerrado de TV

- Contadores de tráfico

- Integración con el uso del suelo y sistema vial.

- Amplia accesibilidad con el pago de una única tarifa

\section{Sustentabilidad:}

$\checkmark$ Ómnibus Híbridos

$\checkmark$ Economía de diésel 35\%

$\checkmark$ Reducción de emisiones contaminantes

Dentro de la Infraestructura, en cada terminal se ha previsto

$\checkmark$ Área de oficinas,

$\checkmark$ Talleres,

$\checkmark$ Surtidores de combustible,

$\checkmark$ Lavadora,

$\checkmark$ Comedores, etc.

Integrador y Operador Tecnológico: Tiene dos funciones principales:

\section{1.- Recaudar}

$\checkmark$ Conciliación de ingresos

$\checkmark \quad$ Liquidación para operadores

$\checkmark$ Atención en taquillas (venta de créditos), etc.

2.- Diseño y optimización de programación de la operación:

$\checkmark$ Definir la programación del servicio de Transporte.

$\checkmark$ Control de la flota:

$\checkmark$ Cumplimiento de itinerarios 
$\checkmark$ Auxilio en caso de emergencia/accidentes

$\checkmark$ Control de maniobras del conductor, etc.

\section{Reglamentos y políticas que lo regulan:}

$\checkmark$ Ley Orgánica de Municipalidades 18.695

$\checkmark$ La Constitución art 118

$\checkmark \quad 19.300$ ley de medio ambiente

$\checkmark$ Ordenanza municipal para recaudar fondos

\section{Obtención de financiamiento:}

$\checkmark$ Empresa Público-Privada

$\checkmark$ Gobierno Regional

$\checkmark$ Ministerio de Hacienda

$\checkmark$ Obras Públicas

$\checkmark$ Concesión de Transporte.

\section{Beneficios de la Política Pública:}

Desarrollo de un transporte sostenible.

$>$ Mayor eficiencia y control.

$>$ Mínima inversión comparada con otros sistemas.

$>$ Construcción de obra en corto tiempo.

$>$ Aumenta seguridad vial, reduce accidentes.

$>$ Mejora la planificación urbana - uso de suelo.

\section{Conclusiones}

La evolución de los sistemas de conectividad vial, conjuntamente con factores de progreso, han dado lugar a la necesidad de presentar esta Política Pública entre las comunas de Temuco y Padre Las Casas, Región de la Araucanía. La lección extraída en los casos anteriormente expuestos, nos ha permitido concluir con la aplicación de un sistema vial para mejorar el servicio público de transporte entre ambas comunas, en busca de la articulación de los diversos actores para la consecución de la eficacia, eficiencia y seguridad, con la visión de alcanzar un bien colectivo. Es importante señalar, la relación que debe existir entre los aspectos institucionales, la autoridad pública y los operadores privados.

\section{Recomendaciones:}

La implementación de Políticas Públicas con participación entre involucrados público-privado, es de de suma relevancia para el desarrollo y crecimiento de una población, en este caso, la propuesta de conectividad vial entre las comunas de Temuco y Padre Las Casas, contribuirá a que los usuarios reduzcan sus tiempos de traslados, incrementen su seguridad y con la reducción de gases contaminantes, mejoren su salud. Con las experiencias demostradas a nivel nacional y en varios países de Latinoamérica, es necesario contar con buses de alta tecnología, los cuales servirán para otorgar un 
servicio eficiente y eficaz. El objetivo de este proyecto, se basará en varias fases que puedan lograr paulatinamente abarcar a muchas comunas de la región de La Araucanía.

\section{Bibliografía:}

Espinoza, S., (2019) "Nuevos desafíos para el transporte público del gran concepción". Diario El Sur. https://www.elsur.cl/impresa/2019/06/10/papel/

Ley $\mathrm{N}^{\circ} 18.695$ Orgánica Constitucional de Municipalidades.

https://www.bcn.cl/leychile/ navegar?idNorma $=251693$

Mantilla, J., Acevedo, H., Duque, C., Galeano, C., Carrión, S., (2008). "Proyección de costos de un bus articulado con motor dedicado a gas natural para ser utilizado en los Sistemas de Transporte Masivo de Colombia".

Mapa Estratégico Seremi de Transporte y Telecomunicaciones, Región de la Araucanía.

http://www.dtpr.gob.cl/mapa_estrategico

Metro Valparaíso S.A., (2009). Implementación Modelo de Integración Bus + Metro.

https://sochitran.cl/static/upload/archivos/sochitran/4201/archivo-adjunto/3/1439234394.pdf

Ministerio de Transporte y Telecomunicaciones. Resolución exenta Nº161

https://www.bcn.cl/leychile/navegar?idNorma $=1115816$

Municipalidad de Padre Las Casas, (2020-2025). "Plan de Desarrollo Comunal". http://www.padrelascasas.cl/plc/INFORME\%20FINAL\%20PLADECO\%20PLC.pdf

Municipalidad de Temuco, (2020-2024). "Plan de Desarrollo Comunal".

https://www.temuco.cl/el-municipio/pladeco/

Pardo, C., (2009). "Los cambios en los sistemas de transporte masivo en las principales ciudades de américa latina”. CEPAL

Pinheiro, C., (2005). "La gestión urbana, Curitiba una experiencia continua en soluciones de Transporte". http://www.planum.net

Valenzuela, E., (1996). "Adiós al municipalismo o la Fecundidad de una red de Gobierno Local".file:/ / C:/Users/angel/Downloads/adiós\%20al\%20municipio\%20red\%20de\%20gob.\%20ev.pd $\mathrm{f}$ 